\title{
Bonding of Structural Parts of Vehicle Bodies and Aspects of Passive Safety
}

J.Kovanda, T.Růžička*

Faculty of Transportation Sciences, Czech Technical University in Prague, Czech Republic

*Corresponding author: tomas.ra@seznam.cz

\author{
M.Kepka \\ Research Centre of Rail Vehicles, Faculty of Mechanical Engineering, University of West Bohemia in Pilsen, \\ Czech Republic
}

\begin{abstract}
Many new materials, technologies and new structural solutions are applied in vehicle bodies. With the application of new, non-conventional materials we obtain better capabilities, a higher level of safety, and, last but not least, weight reduction. At a time when energy savings are sought after, weight reduction is a great motivational factor for the application of composites, aluminium and magnesium alloys, etc. The use of nonconventional materials is connected with requirements for a different technology for the manufacture of vehicle bodies, as well as the issue of mutual connection of individual parts. Completely different actions, which we are not accustomed to in current structures, take place at the boundaries of mechanically, as well as chemically, different substructures. The current pieces of knowledge relate mainly to contemporary materials, or possibly to the classical ways of connecting - welding, screwed joints, and the like. However, we do not completely understand the largely expanding bonded joints, and, in combination with non-conventional materials, we know even less about them. Computer simulations are of no help because the characteristics of these bonds are not described very well. The below-mentioned experiment was focused on this area of structural bonding of composite materials; its objective was to clarify these relations. The actual experiment included a crash test of a vehicle with a body made from composite materials, whose deformation zone had individual elements bonded with various types of structural adhesives. The deformation zone of the experimental body was designed on the basis of the knowledge obtained from tensile tests. The tests were to verify mechanical characteristics of structural joints. Based on the obtained knowledge it was possible to specify the suitability of the use of individual joints and materials in the deformation zone. The outcome of the performed experiment is a judgement of the destruction of individual joints, destruction of the entire body, and fulfilment of passive safety criteria.
\end{abstract}

KEY WORDS: vehicle body, non-conventional material, composites, structural adhesive, boundary relation, passive safety. 


\section{TENSILE TESTS OF BONDED JOINTS}

\subsection{TEST SAMPLES}

19 sets of samples joined using four different adhesives, in combination with six bearing materials (see Figure 1) were defined for tensile tests. The goal was to verify the characteristics of mutual relationships between the materials and general mechanical characteristics. Individual samples were loaded by shear in tension, which is one of the basic loads in real practice.

The following adhesives were selected to bond the samples: Dinitrol F500, Dinitrol PU F500LP, IPS Weld-On SS1505 and joint by overlamination. The selection of these types of adhesives was carried out on the basis of the empirical knowledge of the producers of composite body parts.

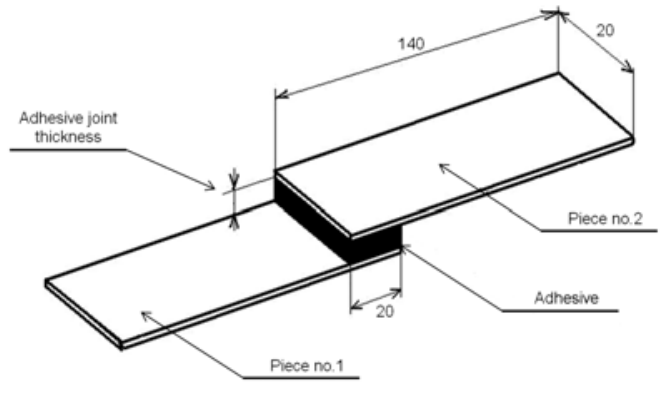

Figure 1: Shape of tensile sample

Dinitrol F500 is a polyurethane adhesive material designed for exposition to high mechanical load. It is a structural, high-strength adhesive. Application of the adhesive is recommended by the manufacturer through a primer Dinitrol 550 Multiprimer.

Dinitrol PU F500 LP is an adhesive on the basis of polyurethane prepolymers. It is again a flexible type of adhesive with high ductility. The difference between it and F500 is in the decreased content of filling - solid particles. Both types of Dinitrol adhesives are one-component adhesives.

IPS Weld-On SS1500 is a compact series of two-component adhesives on the basis of methacrylate. The adhesive is designed for joints under very high mechanical load. This type of adhesive was selected as a representative example of rigid joints, enabling almost no mutual movement of the connected parts.

Overlamination is the last tested joint type. In this joint type, at least one of the bearing parts has to be made from composite material and the other part is applied on it - lapped over. The connection is made by two or more layers of composite that overlap the applied part. To achieve a good connection between the basic - bearing layers of the composite and the overlapping layers, the applied part is perforated. The bearing parts included materials commonly used in vehicles, such as steel plate, aluminium plate, steel plate with galvanic zinc surfacing, steel plate with zinc dipping surfacing, rust-free plate, and glass fibre composite as a representative of composite structures.

\subsection{DEVICES USED FOR LABORATORY TESTS}

An Instron, model 3382, loading machine was used to carry out the tensile tests. It is a device for tensile and compression tests of materials with a working range of up to the force of $100 \mathrm{kN}$. The accuracy of the loading force is $0.5 \%$ and the maximum frequency of data recording $100 \mathrm{~Hz}$. The outcome of the tensile tests was a stress-strain diagram for each tested sample. 


\subsection{RESULTS AND THEIR EVALUATION}

The typical course of the stress-strain diagram of the adhesive Dinitrol F500 is depicted in Graph 1. The course shows linear dependence from the beginning of the tensile test almost up to the strength limit. In the case of some samples non-linearity occurred at approximately 80 to $85 \%$ of maximum strength. The reason for this behaviour is a higher portion of plastic deformation of the adhesive. The adhesive F500 achieves average strength limit values of 4.3 MPa. The values of relative elongation were around $75 \%$. Based on the performed tests it can be stated that the adhesive is suitable for sets of road, as well as rail, vehicle bodies and special superstructures exposed to the largest loads. During the tests, the adhesive showed good adhesion to a wide range of materials (metals even with various surfacing, composites etc.).

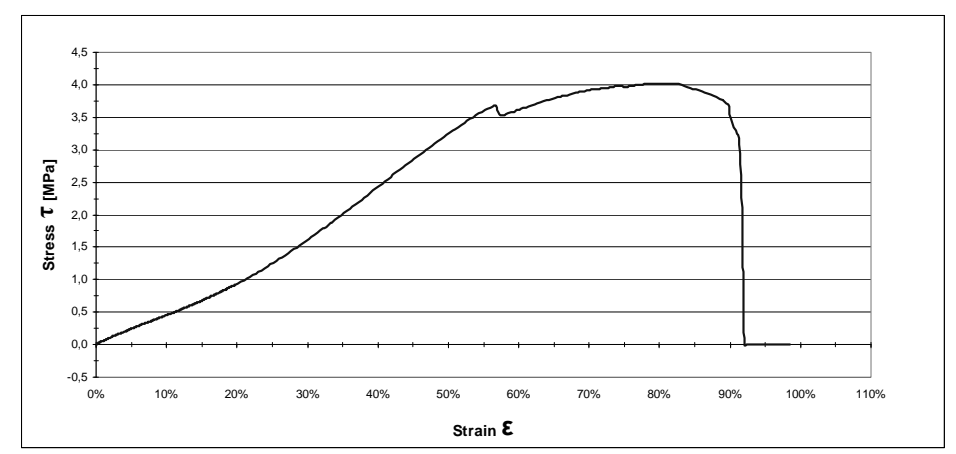

Graph 1: Stress-strain diagram of Dinitrol F500 adhesive

The course of the stress-strain diagram of the adhesive Dinitrol PU F500 LP is linear up to the maximum tensile force (see graph 2). There then occurs destruction and quite immediate loss of strength. The maximum tension reached values of approximately 5.2 MPa. As opposed to the adhesive Dinitrol F500, these joints do not feature elastic deformation in the area of maximum tension. Much better adhesion between the primer, the adhesive and the bearing material was apparent during the analysis of the test samples. The samples with the adhesive PU F500 LP reached values of relative elongation of about $75 \%$, i.e., comparable with the type F500. There were no apparent differences between the stress-strain diagrams from the point of view of different types of bearing materials. Thus, the adhesive has approximately the same behaviour in composite, as well as metal, joints.

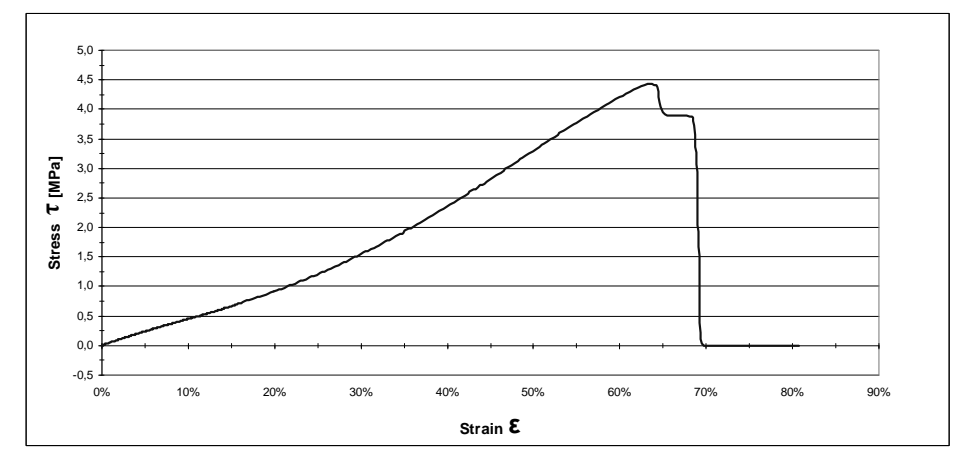

Graph 2: Stress-strain diagram of Dinitrol PU F500 LP adhesive

Samples of joints bonded by the adhesive IPS Weld-On SS1505 behaved much more explicitly during the tensile tests in comparison with all other samples. The strength limit is very easily visible in the course of the stress-strain diagram (see Graph 3). When the strength limit was exceeded, immediate destruction, and hence also loss of strength, occurred. The adhesive reached strength values of 9.5 MPa, which is about twice as much as in the case of Dinitrol adhesives. The adhesive IPS Weld-On SS1505 reached values 
of relative elongation of between 8 and 11.5\%. It is a very rigid bond, which enables almost no mutual movement in the joint. The inclination of the stress-strain diagram is quite steep. The adhesive shows quite good adhesion to the bearing material. Damage of the joint occurred in the material of the adhesive, but also by delamination of the composite. Hence, application of these types of adhesives is suitable for a wide range of structural bonds in vehicles. Advantages of the adhesive IPS Weld-On in comparison with Dinitrol adhesives are in the technological area, as well as the mechanical properties. A primer is not necessary for bonding; strength of the joints under shear load is two to three times higher and also possesses a higher thermal endurance $\left(-55^{\circ} \mathrm{C}\right.$ to $\left.+121^{\circ} \mathrm{C}\right)$. Resistance to chemical exposition is good as well. The adhesives IPS Weld-On are characterized by a combination of high strength and rigidity.

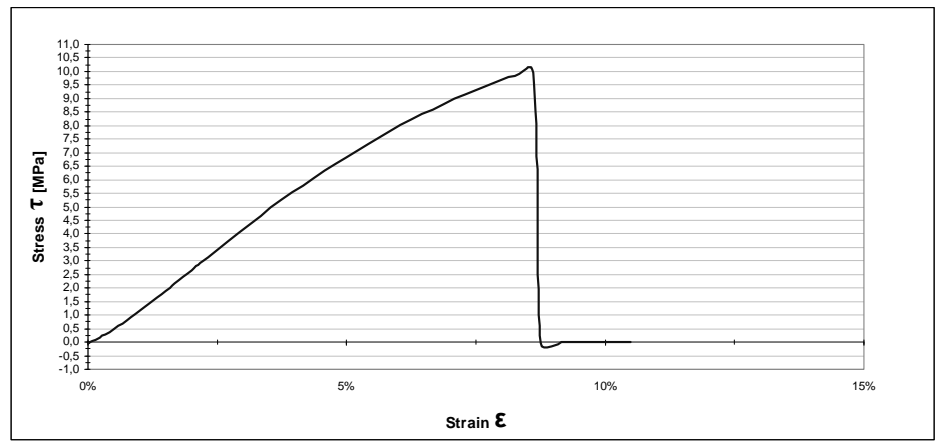

Graph 3: Stress-strain diagram of IPS Weld-On adhesive

The last joint type was overlamination. In this case we are not talking about bonding in the strict sense of a word. Nevertheless, this type of joint is quite often used in technical practice, both in rail vehicles and in bus bodies. The typical course of the stress-strain diagram is hard to determine (see Graph 4) in case of this joint. Destruction of individual composite layers or the ripping off of one of the bearing parts occurs during the tensile tests. The destruction is spread gradually, and thus the course of the stress-strain diagram features several leaps in the strength limits. The strength of this joint is around 3.25 MPa. As regards flexibility it is a very rigid joint with relative elongation of $7.7 \%$, which enables very small mutual movement of parts.

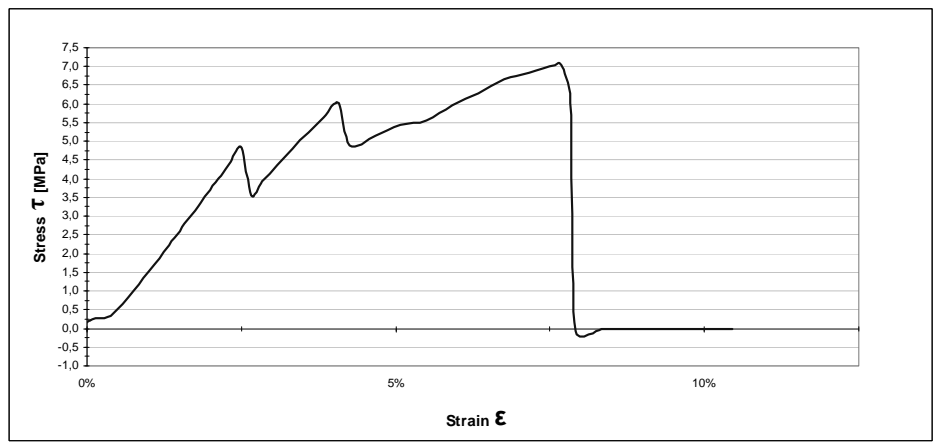

Graph 4: Stress-strain diagram of over-laminated point

\section{DYNAMIC EXPERIMENT - CRASH TEST}

\subsection{CRASH TEST CONCEPTION}

Experimental verification of properties of joints under dynamic load was carried out using a crash test. Joints of structural parts from various materials were put into a vehicle body 
that was designed specifically for this crash test. The evaluation also included an evaluation of the non-aggressiveness of such a vehicle on other crash participants.

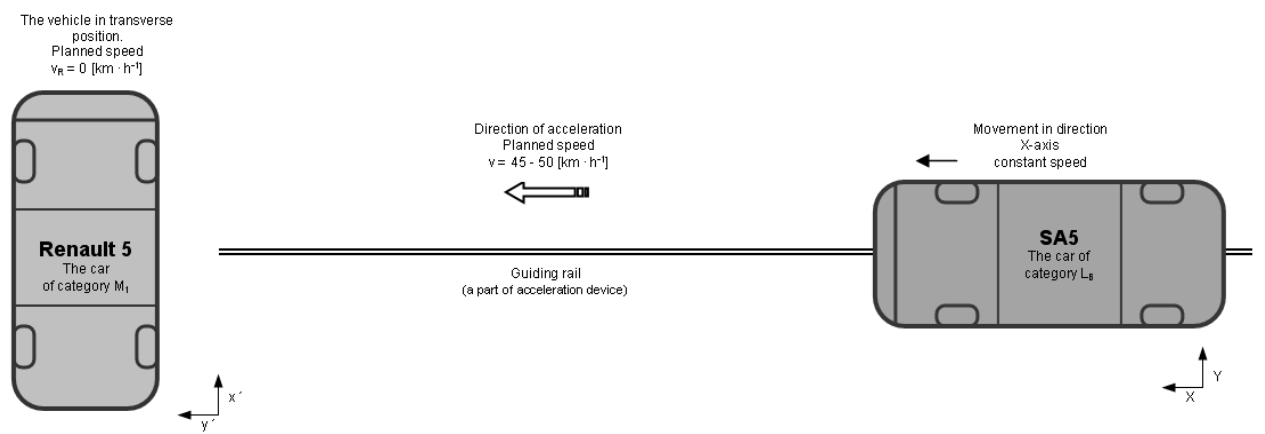

Figure 2: Layout before crash test

The crash test conception was selected to simulate a compatible crash of two vehicles when one of them has not respected the right of way at a crossing in town traffic. The layout before the crash is shown in Figure 2. In the crash test, the main vehicle had all-composite body (hereinafter SA5). Based on the analysis of results of tensile tests and proceeding recommendations, a concept of a deformation zone of this body was proposed. Bonded joints, as well as overlamination, were used in the construction of SA5. The planned speed of the crash was 45 to $50 \mathrm{~km} / \mathrm{h}$. The vehicle was accelerated and directed by a device for the acceleration of vehicles. A manikin dummy was put into the vehicle to evaluate the key indicators of passive safety of the SA5 vehicle and the impact on passengers.

The second crash test participant was the vehicle Renault 5. Before the crash test the vehicle was positioned perpendicularly to the axis of movement of the SA5 vehicle. The speed of Renault 5 at the moment of the crash was zero. The frontal impact of the SA5 vehicle was directed at the "B" pillar of the right side of the Renault 5 vehicle. A child dummy (size P3) in a child seat was placed on the right rear seat. An evaluation of the crash test and factors of passive safety was carried out with the use of records from accelerometers, which were placed on the body of SA5 and measured acceleration in the direction of movement of the axis of the vehicle (X-axis). Furthermore, we placed a three-channel accelerometer to the head of the Manikin dummy; it measured acceleration in the direction of axes $\mathrm{X}, \mathrm{Y}$, and $\mathrm{Z}$. The last accelerometer was placed into the head of the child dummy in the vehicle Renault 5. This sensor recorded acceleration in the direction of the axis $y$ ' of the coordinate system of the vehicle Renault 5, which corresponds with the axis $\mathrm{X}$ of the vehicle SA5.

\subsection{THE MAKING OF THE EXPERIMENTAL BODY SA5}

An all-composite body of a small town vehicle of category $L_{B}$, which was manufactured in small series for Auverland A5 vehicles (designed for town operation with a maximum speed of $50 \mathrm{~km} / \mathrm{h}$ and a $600 \mathrm{ccm}$ engine), was used as a basis for the special body designed for the dynamic test. Only the shell of the body from the original vehicle, made from glass fibre composite, which was designed as self-bearing, was used for the experiment. The floor pontoon, as well as some parts exposed to larger forces (places for the attachment of the engine, attachment of axles, etc.), were reinforced by inserts of aluminium and galvanized plate bonded with the composite by overlamination. All bearing elements and deformation supports, which were able to produce deformation work, were removed from the frontal deformation zone.

These elements were replaced by reinforcements that were made on the basis of knowledge obtained from the tensile tests. In the new version of the deformation elements 
we used flexible types of structural adhesives (Dinitrol), rigid joints (adhesive IPS Weld-On), as well as joints with overlamination.

The conception of the new proposal of the deformation was based on a gradual increase in rigidity of individual elements and joints. Hence, the front part of the body had the character of a progressive deformation zone, which became tougher with the progressing deformation and created higher and higher deformation work. At the beginning of the impact action the rigidity of deformation elements is relatively small; the body should behave favourably when hitting pedestrians, i.e., the body should not be aggressive against the pedestrians.

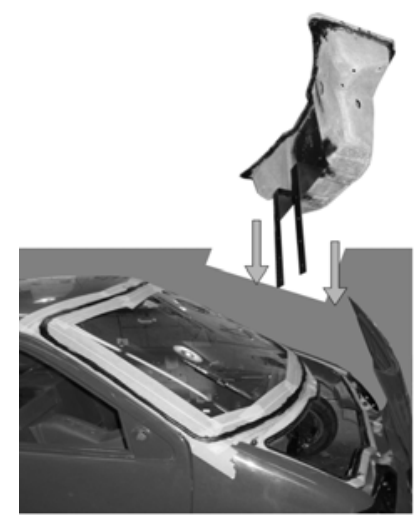

Figure 3: Front boot

The first part of the deformation zone consisted of a front bumper manufactured only from composite material. Composition of the laminate with a total thickness of $4.5 \mathrm{~mm}$ contained $50 \%$ of glass reinforcement thanks to the application of 1 layer of standard fabric and 1 layer of multiaxial fabric.

The second part of the deformation zone was created by integration of a part of the front boot, which is entered into the front part of the body (see Figure 3). It did not have any strength function in the original body. In this case, the part of the boot was bonded around the entire circumference to the body by the flexible adhesive Dinitrol, so the strength of this part participated in the absorption of deformation energy. Three reinforcing elements were placed between the front bumper and a part of the boot. The centre of the body was a laminate "U"-profile of sandwich structure with a thickness of $4 \mathrm{~mm}$, where "coremat" formed the core of the laminate. The bonded area of the bumper was $20,000 \mathrm{~mm}^{2}$ and on the side of the boot it was $25,000 \mathrm{~mm}^{2}$. Reinforcements from steel plate with a thickness of $1.5 \mathrm{~mm}$ were placed on both sides of the body. The reinforcements were bonded to the front bumper profile by a joint area of $19,500 \mathrm{~mm}^{2}$ and to the front part of the boot area by a joint area of $8,000 \mathrm{~mm}^{2}$. The flexible structural adhesive Dinitrol was also used for bonding.

The third deformation zone degree was designed with the application of rigid joints. A steel plate profile was placed between the boot part and the partition separating the area for passengers; on the side of the partition it was screwed on and with the bottom part of the boot it was bonded by overlamination. The overall area of overlamination of both reinforcements was $16,800 \mathrm{~mm}^{2}$. To increase the adhesion of overlamination with the bearing part the steel plate was perforated.

The last structural reinforcement was from composite material reinforcements placed into the boot. These very rigid reinforcements were fit together with the boot to fit as accurately as possible with the surface of the boot walls, and subsequently they were bonded to the walls by the adhesive IPS Weld-On SS1505. The thickness of the laminate in the middle of the reinforcement ribs was $5 \mathrm{~mm}$, and on both ends the ribs were reinforced 
by the so-called head of the rib to a total thickness of $11 \mathrm{~mm}$. The composite was reinforced with glass fabric with a rectangular arrangement.

\subsection{CRASH TEST CONDITIONS}

During the crash test, the vehicle SA5 was accelerated using a pulley device for the acceleration of vehicles, which also performs the leading and self-centring function. The basis of the accelerating device was a sufficiently long rail enabling the acceleration of the vehicle to the specified speed and its stabilization. Another vehicle with sufficient power is used as a source of the traction energy. Inside the rail there is a slider placed on ball bearings.

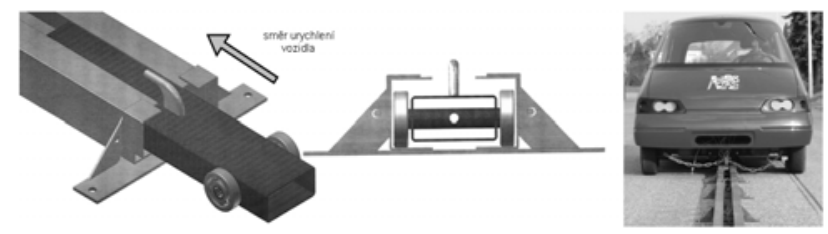

Figure 4: Accelerating rail

The slider was accelerated using a steel cable. The vehicle SA5 was attached to the slider by a chain. At the end of the rail there was a self-locking brake to stop the slider. The accelerated vehicle became unhooked and continued due to inertia for another $1 \mathrm{~m}$ approximately to the point of crash. Figure 4 shows the accelerating device.

\subsection{CRASH TEST EVALUATION}

The impact of the vehicle SA5, whose actual speed was $47 \mathrm{~km} / \mathrm{h}$, moved the vehicle Renault 5 by approximately $3 \mathrm{~m}$ (Note: measured against the centre of the car). Figure 5 shows the final position of the vehicles after the crash test.

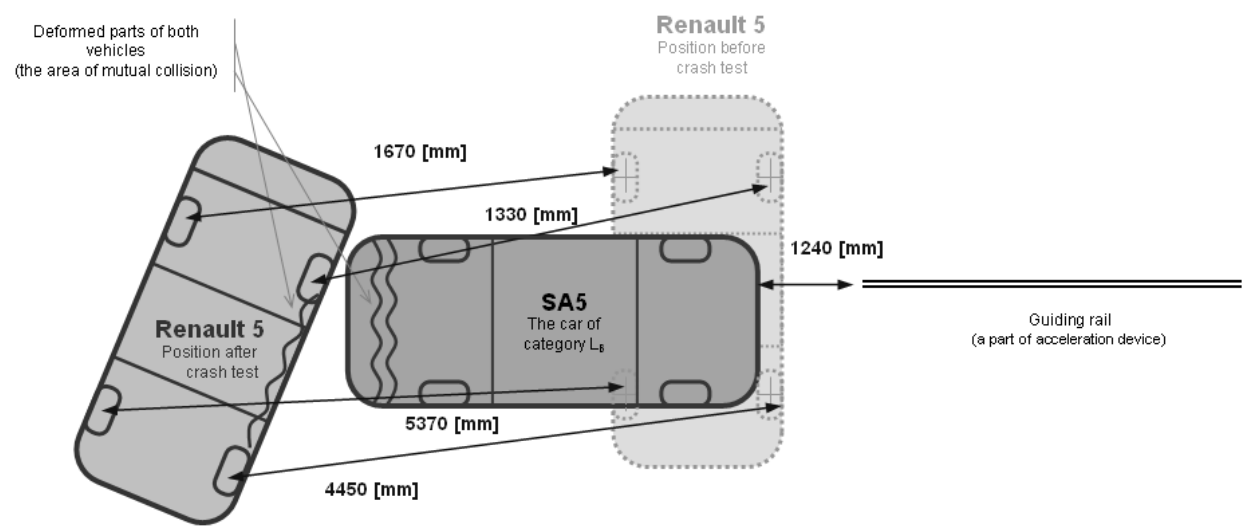

Figure 5: Layout after crash test

Outputs from the accelerometers were connected to the B\&K analyzer, which processed the data. The actual evaluation of courses of acceleration was carried out using the software HyperWorks-HyperGraph. Filters were applied to the actual courses - they removed higher harmonic values from the entire course of acceleration. An evaluation of passive safety on both used dummies using the HIC method (head injury criterion) was carried out using the above-mentioned software. From the accelerometer placed on the body of the vehicle SA5 we obtained the actual course of acceleration of the body in time (see Graph 5). Acceleration values in periods of $10 \mathrm{~ms}$ were read from the graph. Deformations were read from high speed camera records with the same sampling frequency. The deformation force was calculated 
from these data. The dependence of the deformation force on the deformation is depicted in Graph 6. We performed the same calculation of the deformation force and read the deformation values from the high speed camera records for the vehicle Renault 5. The course of the dependence of the force on the deformation is apparent in Graph 7.

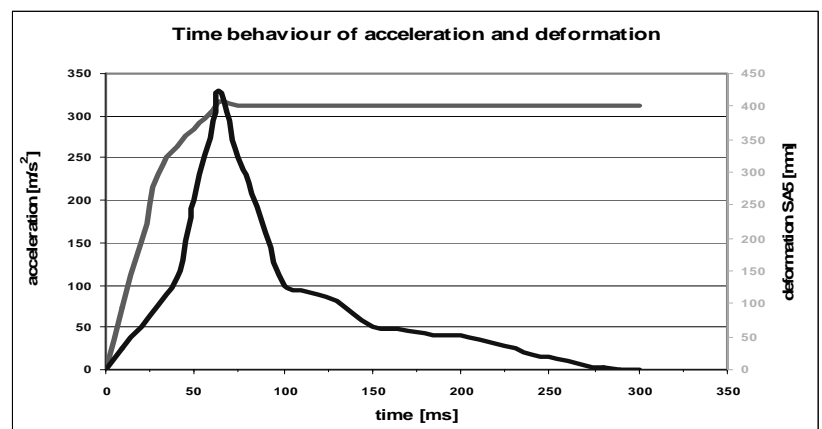

Graph 5: SA5 Acceleration \& deformation

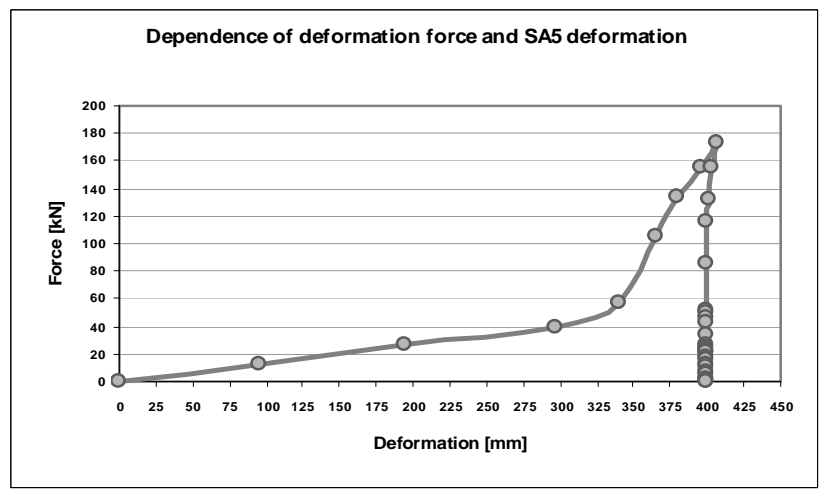

Graph 6: Deformation force of SA5

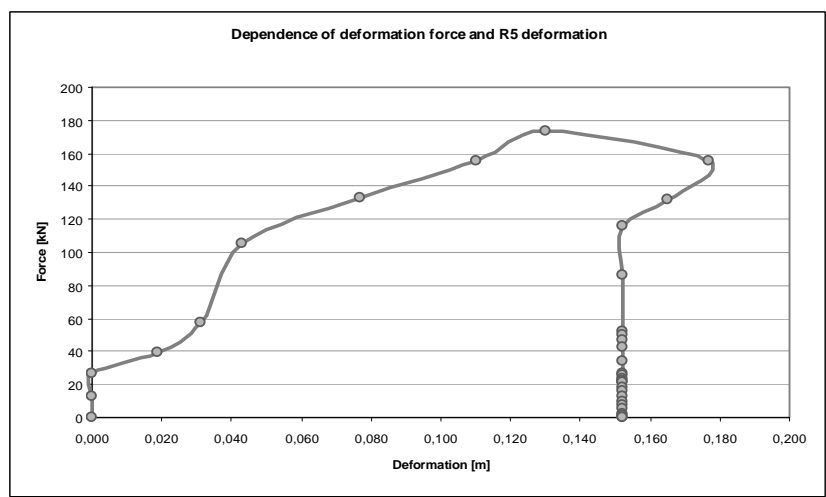

Graph 7: Deformation force of R5

The input energy of the entire set is the kinetic energy of the vehicle SA5. When we simplify it slightly, this energy divides into three main parts - the deformation work of SA5, the deformation work of R5, and the work to move the vehicle R5. The deformation work of both vehicles equals the area below the curve in the graph of the dependence of force on the deformation. By integrating the function $\mathrm{f}(\mathrm{x})$ in a closed interval we obtain the deformation work of each vehicle:

$\mathrm{W}_{\mathrm{SA} 5}=14727.5 \mathrm{~J} ; \mathrm{W}_{\mathrm{R} 5}=20555.4 \mathrm{~J} ; \mathrm{W}_{\mathrm{p}}=9553.3 \mathrm{~J} *$ ) work to move the vehicle $\mathrm{R} 5$. 
The body of the vehicle SA5 deformed smoothly from the front bumper up to the partition of the passenger area. Graph 8, which depicts the course of acceleration measured directly on the solid part of the body, shows gradual degradation of individual reinforcing elements. If simplified slightly, this part of the course could be replaced by a linear course.

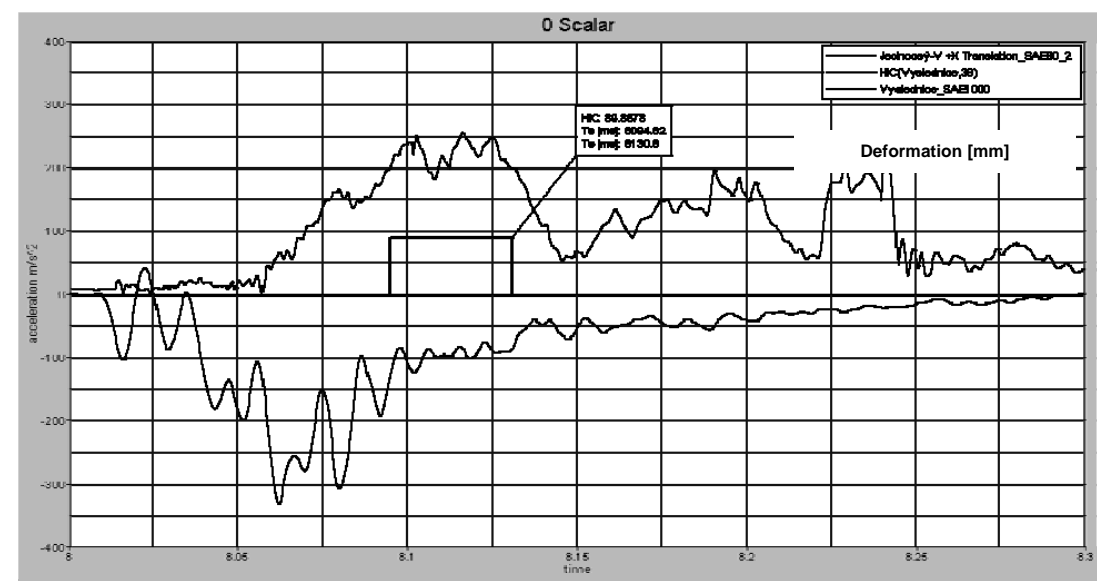

Graph 8:

During the deformation, the axle was bent and the wheels came into contact with the body. Deformation work was thus transmitted to the frame of the body; it spread mostly through the door sills, partition of the passenger area, "A" pillars and " $B$ " pillars. Cracks and other damage were visible at these parts. Nevertheless, the important thing was that no external part penetrated into the passenger area and the passenger area remained completely preserved.

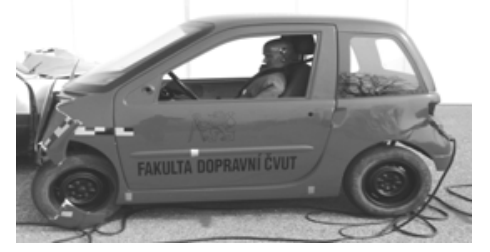

Figure 6: Deformation of SA5

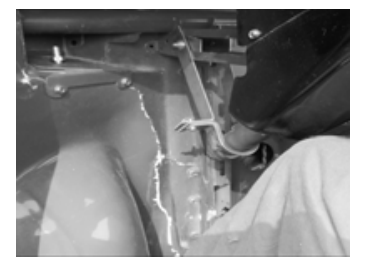

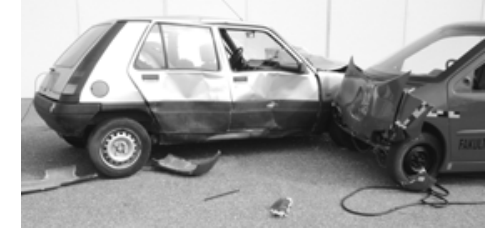

Figure 7: Deformation of R5

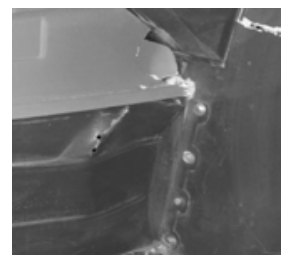

Figure 8 \& 9: Deformation of SA5 interior

One of the main outcomes of this experiment was an evaluation of the composite body of the vehicle SA5 from the point of view of passive safety. We analyzed data from accelerometers placed in the head of the Manikin dummy. Courses of acceleration in the direction of axes X, Y, and Z are depicted in Graphs 9 to 11. The graphs always show two curves. The curve below the $\mathrm{X}$-axis shows acceleration on the solid part of body of the vehicle SA5 and it is provided in the graphs for reference, so that the shift of the course of acceleration of the dummy in comparison with the body is apparent. Graph 8 shows the result of acceleration in the head of the dummy. The red field then depicts the evaluation of the HIC criterion within a period of $36 \mathrm{~ms}$. The calculated value of HIC was 89.85 (the critical value is 1000). The value of maximum acceleration measured on the body 
was $32.8 \mathrm{~m} . \mathrm{s}^{-2}$. Maximum acceleration measured in the head of the dummy was $26 \mathrm{~m} . \mathrm{s}^{-2}$ in direction of the $\mathrm{X}$-axis.

Since a child dummy was placed in a child seat on the right rear seat in the vehicle Renault 5, which was standing crosswise, HIC evaluation was also carried out to deduce how aggressively or friendly the vehicle SA5 behaves to other crash participants.

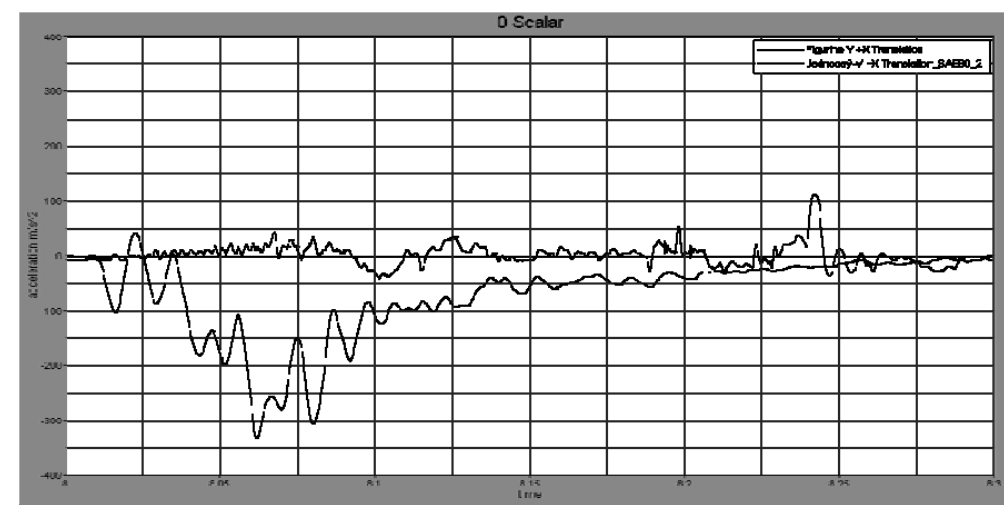

Graph 9: $\mathrm{X}$-axis acceleration of Manikin

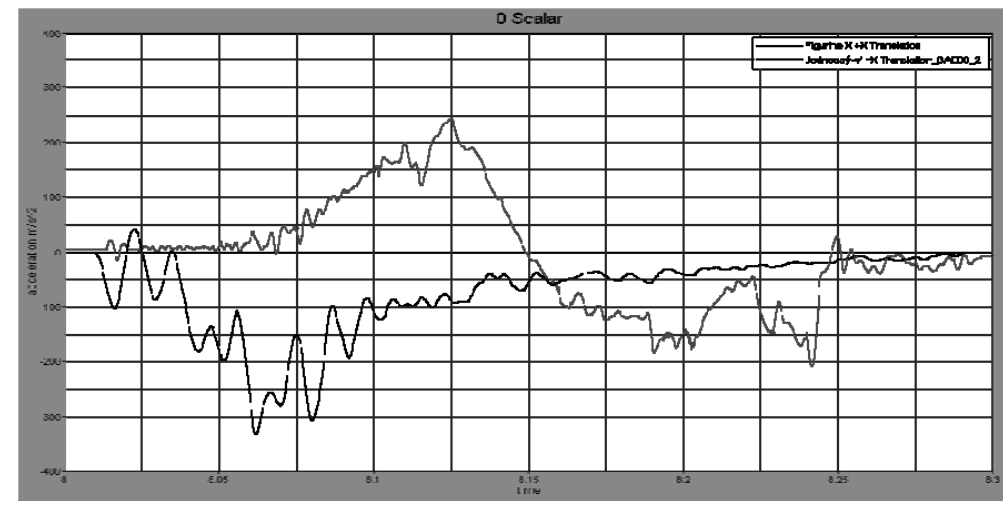

Graph 10: Y-axis acceleration of Manikin

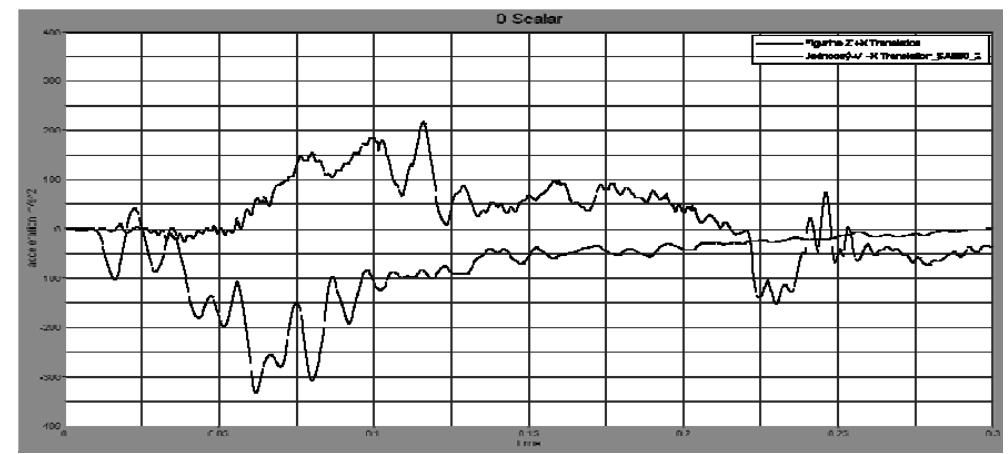

Graph 11: Z-axis acceleration of Manikin

The value of HIC on the P3 dummy was 115.05 (see Graph 12). It is a higher value than in case of the Manikin dummy, but since the limit value is 1000 , we can state a hypothesis that the all-composite body SA5 is friendly towards passengers, as well as towards other crash participants.

\subsection{EVALUATION OF INDIVIDUAL JOINT TYPES AFTER THE CRASH TEST}

From the point of view of destruction of individual joint types on the body, the worst results were achieved by connections made by overlamination. Severe destruction occurred 
at these joints during the crash test. The inserted plate part acted as a separator of individual laminate layers in the composite structure. Delamination, and ripping of the layers above and below the insert (see Figure 10), occurred during the load.

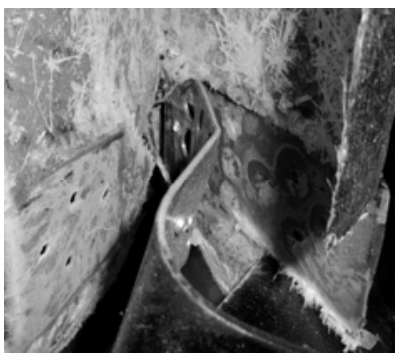

Figure 10

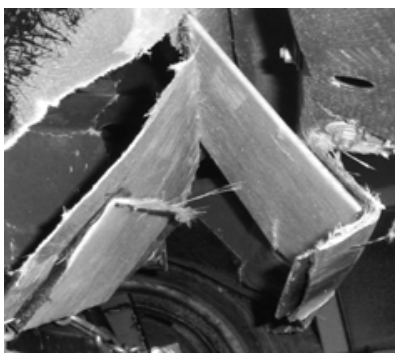

Figure 11

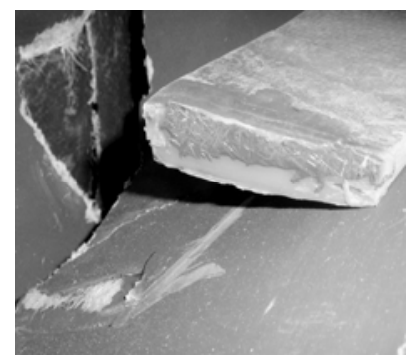

Figure 12

Reinforcements bonded by flexible structural adhesives Dinitrol were not damaged by the crash. The reason was their high elasticity, but also the overdesigning of these joints by approximately $27 \%$. Thus, due to the impact energy there occurred destruction of the actual reinforcements (rupture of the laminate, bending of the steel plate), and the bonded joints remained without damage (Figure 11). As regards the laminate ribs bonded by the adhesive IPS Weld-On SS1505 into the boot area, the portion of the produced deformation work was lower than the original expectation. The reason was the application of the joint to a place where the surface layer of the boot wall had been finished with a gel coat.

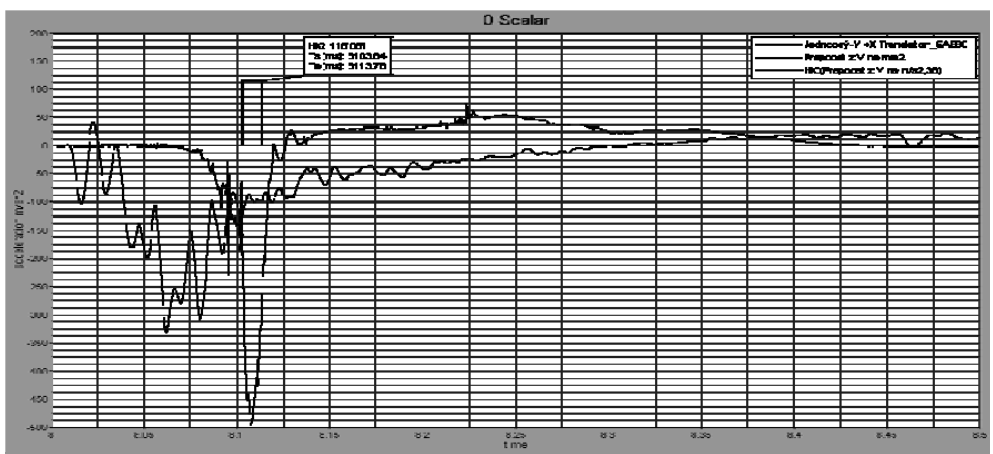

Graph 12:

Its cohesion with other composite layers (matrix, glass reinforcement) is lower, and during the vehicle deformation the rib reinforcements were broken open with a part of the gel coat (Figure 12). Despite this bonded joint not being fully utilized, the portion of the produced deformation work was high, which is indicated by the extent of damage of the surrounding bearing parts of the body.

\section{CONCLUSION}

By generalizing the knowledge obtained from the performed tensile tests and crash experiment it is possible to state several recommendations for the application of bonded joints in vehicle body structures, mainly in combination with composite materials. Polyurethane structural adhesives of Dinitrol F500 type designed for high mechanical load and showing a high degree of relative elongation under tensile load are suitable for application on the structural parts of vehicle bodies, as well as parts of deformation zones, where large deformations can be expected. In the case that the deformation occurs in the areas of elasticity, the adhesive is capable of following the deformed bearing element of the body, while the bonded joint is not degraded in any way and maintains its strength. If plastic 
deformation of body parts occurs, it is good when the adhesive is still elastic and the degradation of joints occurs later on. This effect is supported by a high degree of elongation of the adhesive. The use of polyurethane adhesives is also suitable for the bonding of soffit panels to bearing space frames, for example, and where oscillations occur. These adhesives, thanks to their flexibility and the common thickness of the bonding joint of 2-6 mm, are able to dampen or even completely absorb part of the oscillations.

The analysis of the results of the adhesive Dinitrol PU F500 LP during the experiment confirmed a very good adhesion to the basic materials and a strength of around $15-20 \%$ higher than in the case of F500. It is therefore suitable to use these adhesives for the bonding of windows, for instance. In these applications it can be generally expected that the adhesive will act as a bridge between the body and a very rigid window. Thanks to the combination of strength and flexibility the adhesive does not transmit forces from the body to individual points in the window, but rather spreads the load to a larger area.

The methacrylate adhesives of the type IPS Weld-On SS1505 are suitable due to the large strength of joints for the application on structural bearing elements of vehicle bodies, where a large strength load is expected. On the other hand, the small relative elongation of adhesives needs to be taken into account. The adhesives are not capable of dampening the oscillations. Connections made by these very rigid adhesives are suitable for the last degree of the progressive deformation zone. Besides the bearing parts of vehicle bodies, the application is possible on parts with a small contact area, which serve mainly for the transmission of forces produced by the part itself. For example, attachments of parts in the interior, hinges, etc. Joints made by overlamination are technologically greatly feasible and financially more favourable in comparison with the above-mentioned adhesives. On the other hand, the performed tests showed that the strength, as well as the flexibility, of these joints is very low. This disadvantage can be compensated in practice by enlarging the contact areas of the joints, which brings with it a negative effect - an increase in weight. A connection by overlamination can be used in body parts that are not exposed to such a large load, or in places where pressure loads will be the predominating load on the joint. Overlamination in places with the occurrence of vibrations is not very good either. Thanks to a suitably selected structure, composition of materials, and the applied adhesive it is possible to produce a sufficiently safe deformation zone with a progressive or constant absorption of energy, which will also be considerate of pedestrians.

\section{REFERENCES}

Kovanda, J., Satochin, V.: Passive safety of vehicles, Czech Technical University in Prague.

Bares, R.A.: Composite materials, SNTL, Prague. 1988.

Kovanda, J., First, J., Lenk, J., Micunek, T.: Equipment for vehicle acceleration during dynamic testing of passive safety (VYZ616.005/04), Prague. 2004

Magazines JEC magazine Composites and Automotive News Europe.

\section{ACKNOWLEDGEMENTS}

The contribution was composed in the frame of Ph.D. study of the corresponding author. The authors acknowledge the kind financial support of the Czech Ministry of Transport by the project No. CG711-040-160 and project No.1M0519. Loading machine Instron, model 3382, acquired within the research grant MSM6840770043, AV0Z20710524, was used to carry out the tensile tests. 